\title{
Evaluation of impact ductile fracture behaviour of low carbon austenitic stainless steel SUS304L using damage mechanics model
}

\author{
Hidekazu Takazawa ${ }^{1,2^{*}}$, Kenji Yashirodai $^{1}$, Hiroyuki Yamada $^{3}$, Nagahisa Ogasawara ${ }^{3}$, and \\ Tomohisa Kumagai ${ }^{4}$ \\ ${ }^{1}$ Reserch and Development Group, Hitachi, Ltd. Ibaraki, Japan \\ ${ }^{2}$ Graduate School of Science and Mechanical Engineering, National Defense Academy, Kanagawa, \\ Japan \\ ${ }^{3}$ Department of Mechanical Engineering, National Defense Academy, Kanagawa, Japan \\ ${ }^{4}$ Materials Science Lab., Central Research Institute of Electric Power Industry, Kanagawa, Japan
}

\begin{abstract}
To clarify the applicability of the Gurson-Tvergaard-Needleman (GTN) model for impact ductile fracture behaviour, SHB test was reproduced by finite element analysis (FEA). The strain-rate dependence of the strength for austenitic stainless steel JIS SUS304L was obtained by tensile tests at quasi-static strain rate and impact strain rate. The CowperSymonds power law, which takes into the strain-rate dependence of strength, and the GTN model implemented in the commercial FEA code were used to simulate for impact ductile-fracture behaviour. GTN model parameters were determined by minimizing the difference between the simulated and measured stress-strain curve using response surface method. SHB test was simulated using GTN model obtained from quasi-static tensile test result. Simulation results did not occur the necking and fracture on the specimen. The fracture surfaces were observed by SEM micrograph. The appearance of ductile fracture since dimples are observed, regardless of the strain rate. It is necessary to adjust the parameter to accelerate the nucleation of the void. By identifying the GTN parameters in consideration of the strain rate dependence including impact strain rate. It would be possible to improve the simulation accuracy of impact ductile fracture behaviour.
\end{abstract}

\section{Introduction}

The fracture evaluation method was required to evaluate for structural integrity for infrastructural equipment in the severe accident that exceeds design standards. These requirements were social trends for safety and revised regulatory standards. Therefore, it has become necessary to assess the safety of the materials and the evaluate fractures behaviour of existing structures and their equipment.

As one of the models of damage mechanics applied to the simulation of microscopic ductile-fracture behaviour, the Gurson model, which is based on voids, has been proposed

* Corresponding author: hidekazu.takazawa.te@ hitachi.com 
and applied to damage initiation and propagation within materials [1]. In the Gurson model, a single parameter - it called "void volume fraction" - has a feature that can evaluate the damage initiation and damage propagation of the material. GTN (Gurson-TvergaardNeedleman) model that is based on the Gurson model, considers the influence of nucleation, growth, and coalescence of a void and fracture behaviour was reproduced on simulation [2]. In recent years, a numerical simulation using the GTN model has been used to simulate for ductile-fracture behaviour and assess severe accidents for infrastructure equipment [3]. However, simulation using the GTN model are evaluated for quasi-static fracture behaviour, and there are few evaluation cases for high strain rate deformation.

In this study, in order to clarify the applicability of the GTN model to impact ductile fracture behaviour, SHB test was reproduced by fracture simulation.

\section{Ductile fracture simulation}

\subsection{Test condition}

The strain rate dependence of material strength for austenitic stainless steel Japan Industrial Standards SUS304L [4] was investigated and formulated for impact ductile-fracture simulation. Chemical composition of SUS304L is listed in Table 1. The final solution heat treatment was performed by heating the material at $1060^{\circ} \mathrm{C}$ for 35 minutes and then quenching it in water.

The strain rate dependence of material strength was evaluated by uniaxial monotonic tensile test at quasi-static to impact strain rates. Shape and dimensions of the test specimen is shown in Fig. 1. The tensile tests at quasi-static strain rate $\left(10^{-4}\right.$ and $\left.10^{-2} \mathrm{~s}^{-1}\right)$ were conducted using a universal test apparatus, and at impact strain rate (over $\left.10^{2} \mathrm{~s}^{-1}\right)$ were conducted using a split Hopkinson bar (SHB) tensile test apparatus. Configuration of the SHB tensile test apparatus is shown in Fig. 2. To increase the duration time of elastic wave, input and output bars have long structure. The specimen was unified in a form conforming to JIS Z 2205:2019 [5]. The effect of strain rate on the stress-strain relationship was formulated on the basis of the Cowper-Symonds power law [6]. It is a constitutive equation in which the viscous part of flow stress is proportional to the power of strain rate. In this study, the Cowper-Symonds parameters were determined as $D=284493159$ and $m=0.118$.

Table 1. Chemical composition of SUS304L (mass \%)

\begin{tabular}{|c|c|c|c|c|c|c|c|}
\hline $\mathrm{C}$ & $\mathrm{Si}$ & $\mathrm{Mn}$ & $\mathrm{P}$ & $\mathrm{S}$ & $\mathrm{Ni}$ & $\mathrm{Cr}$ & $\mathrm{Fe}$ \\
\hline 0.01 & 0.52 & 0.92 & 0.021 & 0.005 & 10.11 & 18.32 & Bal. \\
\hline
\end{tabular}

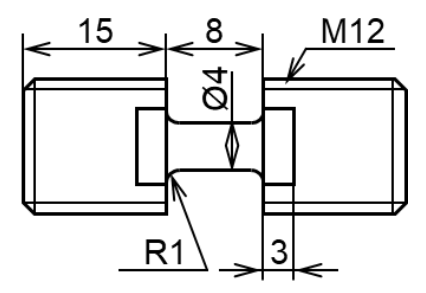

Fig. 1. Shape and dimensions of tensile test specimen (unit: $\mathrm{mm}$ ) 


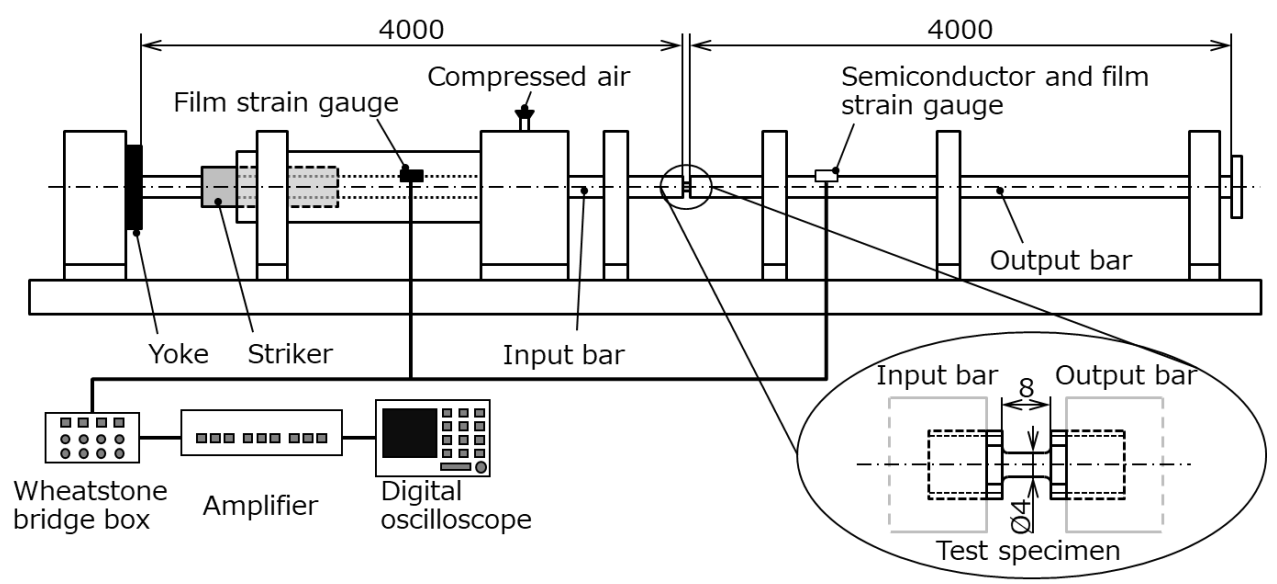

Fig. 2. Schematic of split Hopkinson bar tensile test apparatus (unit: $\mathrm{mm}$ )

\subsection{Damage mechanics model}

The GTN model is given as

$$
\phi=\left(\frac{q}{\sigma_{Y}}\right)^{2}+2 q_{1} f^{*} \cosh \left(-\frac{3}{2} \frac{q_{2} p}{\sigma_{Y}}\right)-\left(1+q_{3} f^{* 2}\right)
$$

where $\phi$ is yield function of the GTN model; $q$ is macroscopic stress including voids; $\sigma_{Y}$ is equivalent stress; $\mathrm{p}$ is hydrostatic stress; $q_{1}, q_{2}$, and $q_{3}$ are constants proposed by Tvergaard; and function $f^{*}$ models the rapid loss of stress-carrying capacity that accompanies coalescence of voids. This function is defined in terms of void volume fraction as

$$
f^{*}=\left\{\begin{array}{cl}
f & \text { for } f \leq f_{C} \\
f_{C}+\frac{\bar{f}_{F}-f_{C}}{f_{F}-f_{C}}\left(f-f_{C}\right) & \text { for } f_{C}<f<f_{F} \\
\bar{f}_{F} & \text { for } f \geq f_{F}
\end{array}\right.
$$

where $f_{C}$ is a critical value of void volume fraction, and $f_{F}$ is the value of void volume fraction which the stress-carrying capacity in the material is completely lost. Rate of increase of void volume fraction is expressed by the summation of two terms, one for nucleation of new voids and one for growth of existing voids, as

$$
\dot{f}=(1-f) \dot{\varepsilon}_{m}^{p l}+\frac{\dot{\varepsilon}_{e q}^{p l} f_{N}}{s_{N} \sqrt{2 \pi}} \exp \left[-\frac{1}{2}\left(\frac{\varepsilon_{e q}^{p l}-\varepsilon_{N}}{s_{N}}\right)^{2}\right]
$$

where first term is change due to growth of existing voids, and second term is change due to nucleation of new voids. Growth of existing voids is based on the law of conservation of mass and is expressed in terms of the void volume fraction. $\dot{\varepsilon}_{e q}^{p l}$ is equivalent plastic strain 
rate. The normal distribution of nucleation strain has mean value $\varepsilon_{N}$ and standard deviation $s_{N}$, and $f_{N}$ is volume fraction of the nucleated voids. The voids are nucleated only in a tensile condition. To simulate ductile-fracture behaviour with the GTN model, these parameters were identified by using the tensile test result.

L18 orthogonal-table simulation based on Taguchi's method was conducted to identify the GTN model parameters. Parameters were determined by minimize the difference between the simulated and measured nominal stress and nominal strain curve at quasi-static strain rate $\left(10^{-4} \mathrm{~s}^{-1}\right)$ using response surface method. The identified parameters of the GTN model are listed in Table 2. Figure 3 shows the nominal stress and nominal strain curve obtained from the tensile test are compared with the simulation results obtained by the GTN model at the strain rate of $10^{-4} \mathrm{~s}^{-1}$. Simulated stress and strain curve with the GTN model is good agreement with the measured result. These parameters were used to the SHB test simulation.

Table 2. Identified GTN parameters using quasi-static speed tensile test result

\begin{tabular}{|c|c|c|}
\hline Symbol & Name & Value \\
\hline$q_{1}$ & Coef. of void volume fraction and hydrostatic pressure 1 & 0.94 \\
\hline$q_{2}$ & Coef. of void volume fraction and hydrostatic pressure 2 & 0.82 \\
\hline$q_{3}$ & Coef. of void volume fraction and hydrostatic pressure 3 & 0.88 \\
\hline$f_{C}$ & Critical void volume fraction & 0.49 \\
\hline$f_{F}$ & Failure void volume fraction & 0.58 \\
\hline$\varepsilon_{N}$ & Mean void nucleation strain & 0.71 \\
\hline$s_{N}$ & Standard deviation of the normal distribution of nucleation strain & 0.11 \\
\hline$f_{N}$ & Void volume fraction of nucleating particles & 0.10 \\
\hline
\end{tabular}

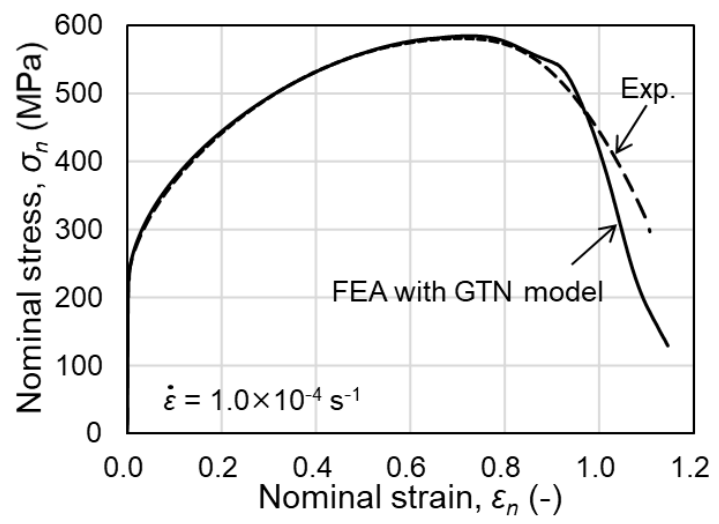

Fig. 3. Comparison between measured and simulated result obtained using GTN mode

\subsection{SHB test simulation}

The FE mesh of SHB test simulation is shown in Fig. 4. It is shown to enlarge the specimen part. The FEA model was a half model based on the axisymmetric properties. An initial 
imperfection of diameter was introduced into the gauge-length area. Dynamic fracture simulation was performed with the commercial FEA code Abaqus ${ }^{\circledR} /$ Explicit (ver.2019).

The nominal stress and nominal strain curve obtained from the SHB test is compared with the simulation results obtained using the GTN model and Cowper-Symonds power law is shown in Fig. 5. There is a difference between the test result and the simulation results. Simulation results using GTN parameter obtained from quasi-static tensile test result did not occur the necking and fracture on the specimen. This indicates that it is not possible to reproduce the impact ductile fracture by a parameter determined by quasi-static test. Although Equation 3 has a strain rate term, void ratio did not increase with increasing strain rate when the analysis results were investigated in detail.

Therefore, the damage criterion of critical value $f_{C}$ and stress-carrying capacity $f_{F}$ were changed to reproduce the impact ductile fracture. At the impact strain rate, fracture occurred by multiplying the damage criterion by 0.01 times when the damage criterion was gradually reduced. However, necking did not occur on the simulation results. This is because that the growth and nucleation voids were not enough for fracture even if the value of damage criterion is changed.

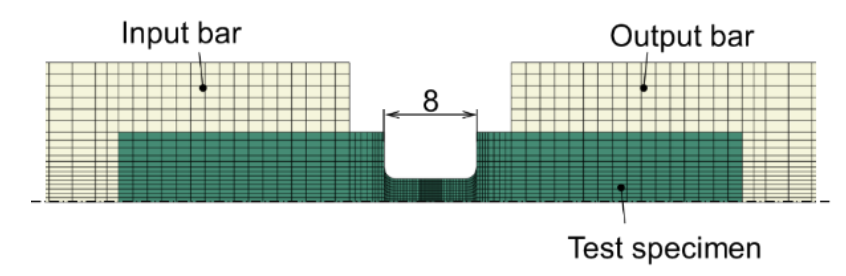

Fig. 4. FE mesh of SHB test simulation (unit: $\mathrm{mm}$ )

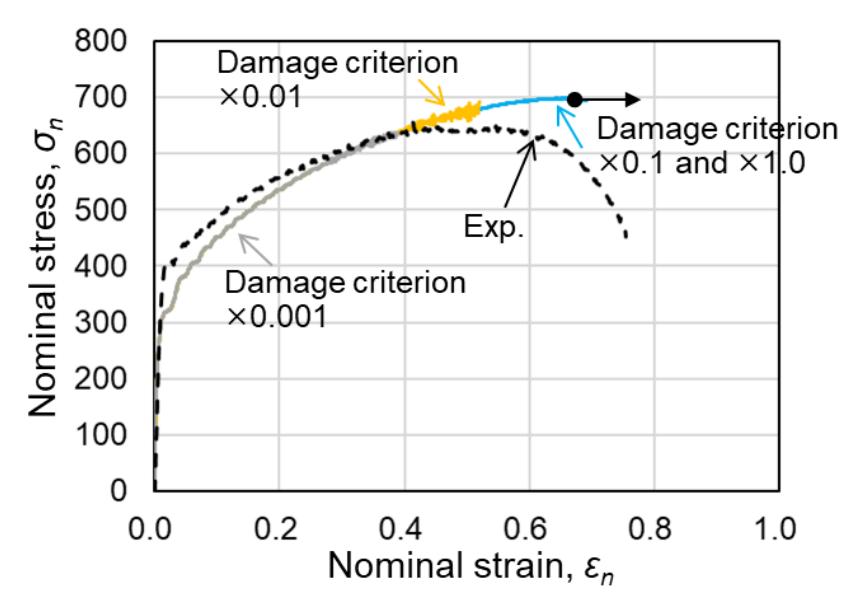

Fig. 5. Comparison between measured and simulated result using GTN model for SHB test

\section{Effect of strain rate on fracture morphology}

In order to investigate the effect of strain rate on fracture morphology, the fracture surfaces were observed by scanning electron microscope (SEM). Figure 6 shows SEM micrographs of the fracture surfaces at the quasi-static $\left(10^{-4} \mathrm{~s}^{-1}\right)$ and impact strain rates. SUS304L shows the appearance of ductile fracture since dimples are observed, regardless of the strain rate. It should be noted that the shape of the dimples at the impact strain rate was larger than the quasi-static strain rate. This indicates that the growth of voids is affected by the strain rate. 
Therefore, it was not appropriate to adjust the damage criterion parameters at the impact strain rate. It is necessary to adjust the parameter to accelerate the nucleation of the void. By identifying the GTN parameters in consideration of the strain rate dependence including impact strain rate, it was predicted that it would be possible to improve the simulation accuracy of impact ductile fracture behaviour.

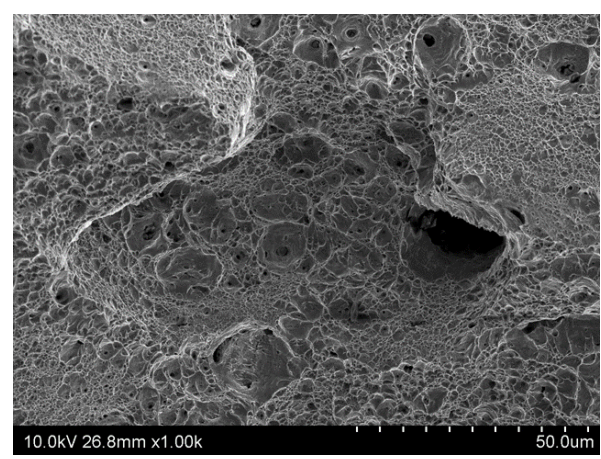

(a) Quasi-static $\left(10^{-4} \mathrm{~s}^{-1}\right)$

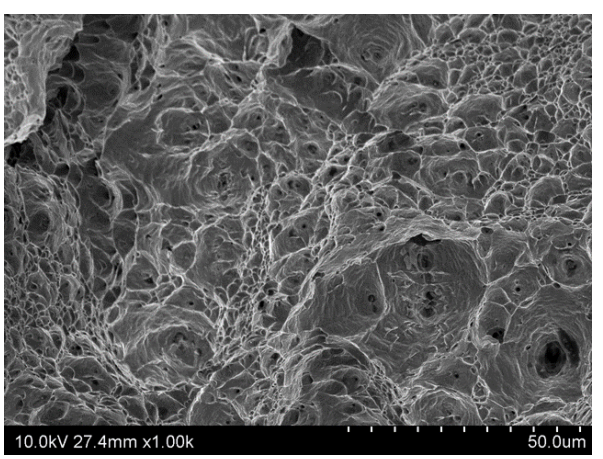

(b) Impact

Fig. 6. SEM micrographs of the fracture surfaces at the quasi-static and impact strain rates

\section{Summary}

In order to clarify the applicability of the GTN model to impact ductile fracture behaviour, SHB test of SUS304L specimen was reproduced by impact ductile fracture simulation. The results are summarized as follows.

(1) The impact ductile fracture simulation using GTN model with the parameters identified by the result of quasi-static strain rate did not occur the necking and the fracture behaviour on the specimen.

(2) SUS304L specimens showed ductile fracture with dimples at both quasi-static and impact strain rates. However, the shape of the dimples at impact strain rate is larger than that quasi-static strain rate.

(3) From the results of this study, in order to evaluate the effect of strain rate on fracture using GTN parameters, how to express void growth by analysis is a future issue.

\section{References}

1. A. L. Gurson, J. Eng. Mater. Technol., 99, pp.2-15, (1977)

2. V. Tvergaard, A. Needleman, Int. J. Fract., 37, pp.197-215, (1988)

3. H. Nakamura, M. Kikuchi, M. Ozawa, R. Kojo, A. Hotta, H. Moritani, and S. Horino, Secretariat of Nuclear Regulation Authority, (2019).

4. JIS, Japanese Industrial Standards Association, Standard No. JIS G 4304:2012, (2012).

5. JIS, Japanese Industrial Standards Association, Standard No. JIS Z 2205:2019, (2019).

6. G. R. Cowper, P. S. Symonds, Brown University Division of Applied Mathematics Report, 28, (1958) 